\title{
Various Types of Image Noise and De-noising Algorithm
}

\author{
Gourav \\ CGC-COE/ CSE, Landran, Mohali, Punjab \\ E-mail: chalotragourav@gmail.com \\ Tejpal Sharma \\ CGC-COE/ CSE, Landran, Mohali, Punjab. \\ E-mail: tejpal.coecse@cgc.edu.in
}

\begin{abstract}
Image de-noising is a procedure that used to upgrade the picture quality after corrupted by the noise. There are a few techniques have been proposed for picture de-noising. Noise lessening and reclamation of image is relied upon to enhance the subjective review of a picture and the execution criteria of quantitative picture examination systems Digital picture is slanted to an assortment of commotion which influences the nature of picture. The criteria of the commotion expulsion issue rely on upon the noise sort by which the picture is defiling. To diminish the image commotion a few sorts of direct and non strategies separating methods and denoising calculation have been proposed. Straight channels are not ready to successfully take out motivation commotion as they tend to obscure the edges of a picture. Then again non straight channels are suited for managing drive commotion. Diverse methodologies for decrease of commotion and image upgrade have been viewed as, each of which has their own restriction and favorable circumstances.
\end{abstract}

Index Terms-Image noise, types of noise, filters, denoising algorithm.

\section{INTRODUCTION}

Advanced image assumes a critical part in our everyday life and in the zone of research and technology. When the computerized image is transmitted starting with one place then onto the next place, during the transmission noise is included into the image. Any type of flag preparing having picture as an info and yield is called picture handling. Because of the defect of the instruments utilized as a part of the picture preparing, noise can be produced . The obstruction amid the transmission corrupt the data. Noise can likewise be created by the transmission blunder and compression. Different sorts of commotions are presented by various commotion sources like dull current commotion is because of the thermally produced electrons at sensor destinations. . Noise corrupts the picture quality that is the reason there is a need to de-noise the picture to reestablish the nature of image. There are different strategies that are help to expel the commotion from the computerized image. But choosing the proper technique is assumes significant part in getting the coveted image. The strategies used to de-noise the satellite picture and medicinal picture are different, therefore the picture denoising strategy utilized for satellite picture is not appropriate for de-noising the therapeutic picture. Noise is presented in the image during image transmission. The additional noise will be of different sorts like added substance irregular commotion (Gaussian clamor), salt and pepper noise, and so on. Contingent upon the kind of the commotion, the corruption of the image will shift. As indicated by the rate of picture quality corruption, the commotion expulsion systems must be picked. The conventional strategies for noise expulsion incorporate NLM channel, Total Variation (TV) technique, Shrinkage models and diverse changes. The wavelet change, curvelet change and wave iota change are the productive changes for picture de-noising calculations. A portion of the strategies are utilizing fluffy rationale and different apparatuses. The majority of the noise expulsion methods proposed till now depend on what sort of commotion is presented. Additionally the application, to which the picture and video are to be utilized, chooses the required commotion evacuation calculation [1]. Noise is the most irritating issue in picture preparing. Noise can be presented by transmission blunders and pressure. Noise is the undesirable impacts created in the picture. Amid picture procurement or transmission, a few elements are in charge of presenting noise in the picture. Contingent upon the sort of aggravation, the noise can influence the picture to various degrees. For the most part our concentration is to expel certain sort of clamor. Hence, de-noising is frequently an important and the initial step to be taken prior to the picture information is broke down It is important to apply a productive de-noising procedure to adjust for such information debasement. One approach to dispose of this issue is the improvement of such a hearty calculation, to the point that can play out the preparing assignments in nearness of commotion. The other route is to plan a filtration procedure to dispose of the commotion from pictures [2] while safeguarding its components, edges and points of interest. Salt and pepper noise is a type of noise generally observed on images. It 
exceptionally speaks to itself as arbitrarily happening white and dark pixels. A successful clamor decrease approach for this kind of commotion includes the utilization of a middle channel or a complexity symphonic mean channel. Salt and pepper noise influences into pictures in circumstances where the picture is exchanged rapidly. Image de-noising has been a very much examined issue in the field of picture handling. However specialists keep on focusing consideration on it to better the present cutting edge. Image de-noising is a critical image handling assignment, both as a procedure itself, and as a segment in different procedures [3]. A lot of approaches to noise reduction a picture or an arrangement of information exists. The primary properties of a decent image de-noising model are that it will expel commotion while saving edges. The point of these techniques is to distinguish edges and subtle elements by methods for neighborhood measurements and smooth them not as much as whatever remains of the image to better safeguard their sharpness. Nonetheless, these strategies normally recognize Impulses as subtle elements or edges to be safeguarded, and, along these lines, they are not ready to lessen that this present framework assess the execution of the picture de-noising techniques.

\section{SourCes OF NoISE In Digital IMAGES}

Noise is measured by the rate of pixels which are ruined. Undermined pixels are either set to the greatest esteem or have single bits. There are a few ways that commotion can be brought into a picture, contingent upon how the picture has been made [6]. For instance:

- If an image is checked from a photo made on film, the film grain is a wellspring of noise. Noise may likewise be the consequence of harm to the film, or be presented by the scanner itself.

- If an image is gained specifically in a computerized arrange, the system for social affair the information, (for example, a CCD indicator) can present noise.

- Electronic transmission of picture information can make noise.

\section{A. Types of noise}

Noise is undesirable or bends flag that may degenerate the quality or the innovation of the image. Commotion has been delivered in the image because of transmission. Along these lines, the primary wellspring of picture denoising is Image Digitization. In image handling, noise delivers an image that may comprise of uneven lines, obscured protest, twisting of pixels, corners, foundation scenes and so forth. Image are undermined because of different sorts of commotions in which some of them are Gaussian, photograph electronic, motivation noise [4].

\section{i. Gaussian noise}

Gaussian noise is otherwise called enhancer commotion or electronic commotion as it produces from intensifier or indicator. It utilizes Gaussian appropriation i.e. typical conveyance. Gaussian noise is a measurable noise having Probability Density Function (PDF) which is equivalent to Gaussian appropriation. This noise is added substance in nature. Gaussian noise is autonomous at every pixel and flag intensity. It is brought about by warm noise. The mean of every pixel of a picture that is influenced by Gaussian commotion is zero. It implies that Gaussian clamor quality influences every last pixel of a picture. A special case is white Gaussian noise, in which the values at any pair of times are identically distributed and statistically independent (and hence uncorrelated). In communication channel testing and modeling, Gaussian noise is used as additive white noise to generate additive white Gaussian noise. The likelihood circulation capacity of Gaussian noise is ringer formed [5]. Communication channels can be affected by wideband Gaussian noise coming from many natural sources, such as the thermal vibrations of atoms in conductors, shot noise, black body radiation from the earth and other warm objects, and from celestial sources such as the Sun. An image with Gaussian noise is shown below:

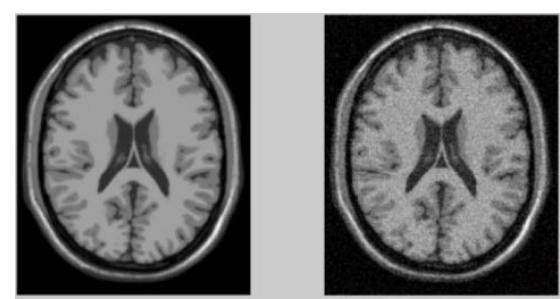

Fig.1. Example of Gaussian noise [1]

Gaussian noise is regularly characterized as the commotion with a Gaussian adequacy dispersion, which expresses that nothing the relationship of the noise in time or the unearthly thickness of noise. Gaussian noise is generally said as repetitive sound portrays the connection of commotion. Gaussian noise is infrequently compared to be of white Gaussian noise, yet it may not necessarily the case. It is added substance in nature, every pixel is free and flag force of every pixel is likewise autonomous. Therefore, every single pixel of an image influenced i.e. an image influenced by Gaussian commotion is zero [5].

\section{ii. Salt and pepper noise}

Salt and pepper noise is otherwise called Impulse commotion, spike noise, arbitrary noise or free commotion. The purposes for the event of this noise are the sharp and sudden changes of image flag, by memory cell disappointment, by breaking down of cameras sensor cells, by synchronization mistakes in image digitizing or transmission [6]. 

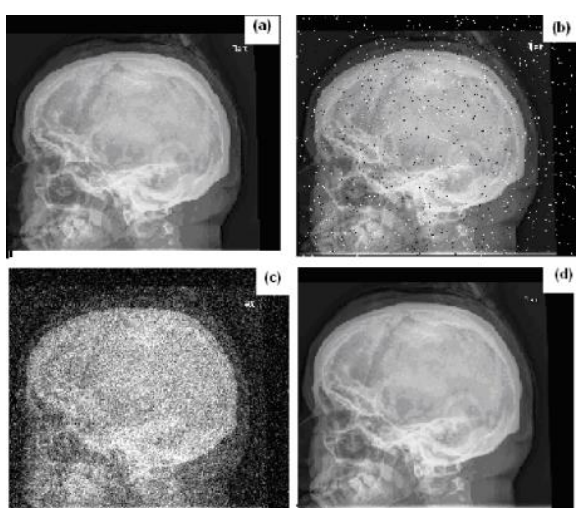

Fig.2. Image with salt and Pepper Noise [5]

Clean molecule in image securing source or overheated defective part can likewise precipitate this sort of commotion. In this sort of commotion, the image is ruined because of high contrast spots on it, having dull pixels in splendid districts and brilliant pixels in dim locales. High contrast speck in an image have some noise esteem i.e. 0 and 1 separately. Commotion esteem for dark is extraordinary low and for white is outrageous high. For 8-bit picture the run of the mill an incentive for 255 for salt -noise and pepper commotion is 0 .

Reasons for Salt and Pepper Noise:

- By memory cell disappointment.

- By failing of camera's sensor cells.

- By synchronization mistakes in image digitizing or transmission.

\section{iii. Poisson noise}

Poisson noise is likewise named as shot noise. It takes after Poisson dispersion which is like Gaussian circulation. It showed up on the picture because of the factual way of electromagnetic waves. This sort of commotion happens when the quantities of photons that are caught with the sensors are insufficient to identify factual changes in estimation. Vacillations of photons are the fundamental reason of Poisson noise. Poisson or shot photon noise is the commotion that can bring about, when number of photons detected by the sensor is not adequate to give recognizable factual information. An image with Poisson noise is given below:

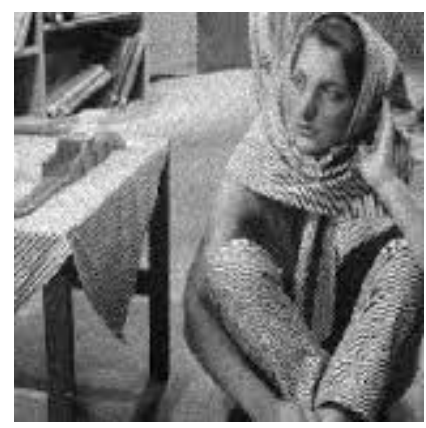

Fig.3. Example of Poisson Noise [6]

This noise has root mean square esteem relative to square root force of the picture. Distinctive pixels are endured by free noise values. At commonsense grounds the photon clamor and other sensor based commotion degenerate the flag at various extents the presence of this noise is seen because of the factual way of electromagnetic waves, for example, x-beams, obvious lights and gamma beams. The $\mathrm{x}$-beam and gamma beam sources discharged number of photons per unit time [5]. These beams are infused in patient's body from its source, in medicinal $\mathrm{x}$ beams and gamma beams imaging frameworks. These sources are having arbitrary variance of photons. Result assembled image has spatial and fleeting arbitrariness. This commotion is likewise called as quantum (photon) image or shot noise.

\section{B. Filters}

Filtering in an image preparing is a premise capacity that is utilized to accomplish many errands, for example, clamor lessening, addition, and re-examining. Sifting image information is a standard procedure utilized as a part of all image handling frameworks. The decision of channel is controlled by the way of the errand performed by channel and conduct and kind of the information. Denoising is important and the initial step to be taken before the pictures information is dissected. It is important to apply a productive de-noising system to adjust for such information corruption. We utilized numerous methods to expel the clamor from the advanced picture. Channels are utilized to expel noise from advanced image while keeping the points of interest of image safeguarded is a fundamental piece of image preparing [6].Filters can be described by different categories:--

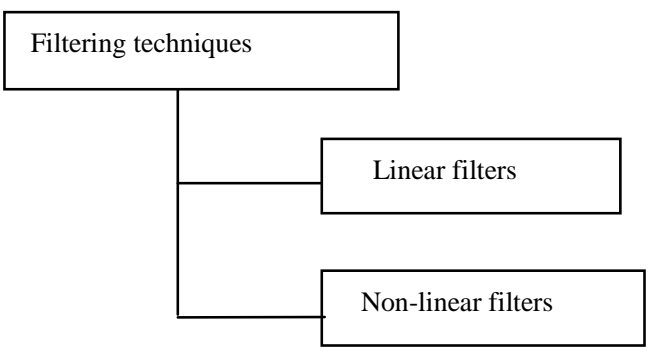

Fig.4. Types of filtering techniques [6]

i). Different Type of Linear and Non-Linear Filters:

a) Mean Filter:

The mean filter is a straightforward filter .It is a sliding-window channel that replaces the middle an incentive in the window. It replaces with the normal mean of all the pixel values in the part or window. Mean filter follows up on an image by smoothing it. The mean filter is a straightforward sliding window spatial filter that replaces the middle an incentive in the window with the normal of all the neighboring pixel values including it. This procedure is rehashed for all pixel values in the picture. By doing this, it replaces pixels that are unrepresentative of their environment. The window is normally square yet it can be of any shape. The window is generally square however it can be of any shape [6]. 


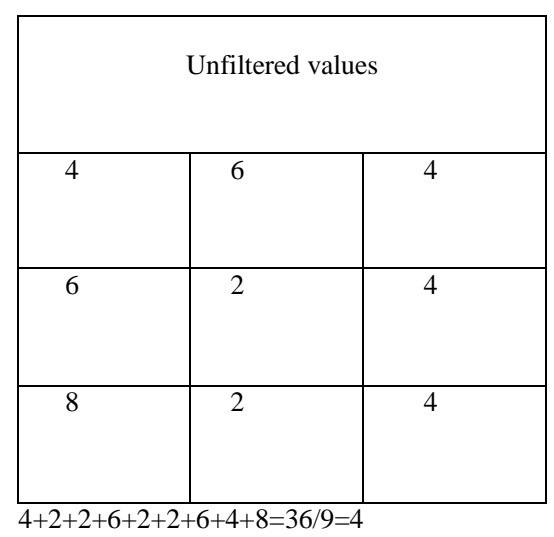

Fig.5. Example of Mean Filtering of a 3x3 Kernel

\begin{tabular}{|l|l|l|}
\hline \multicolumn{3}{|c|}{ Mean filtered } \\
\hline$*$ & $*$ & $*$ \\
& & \\
\hline$*$ & 4 & $*$ \\
& & \\
\hline$*$ & $*$ & $*$ \\
& & \\
\hline
\end{tabular}

Fig.6. Center Value Which is Previously 2 in the Unfiltered Value Is Replaced by the Mean of All Nine Values that is 4 .

Merits:

- Easy to execute.

- Used to evacuate the driver noise.

Demerits:

- It doesn't save subtle elements of an image. A few subtle elements are expels of an image with utilizing the mean channel.

\section{b) Median Filter:}

Median [4] Filter is a basic and effective non-straight channel which is based request insights. It is anything but difficult to actualize strategy for smoothing image. Median filter is a best request static, non-straight filter, whose reaction depends on the positioning of pixel qualities contained in the channel area. Median filter is very prominent for decreasing certain sorts of noise. Here the inside estimation of the pixel is supplanted by the middle of the pixel values under the channel area. Median filter is useful for salt and pepper commotion. These channels are broadly utilized as smoother for picture handling, and additionally in flag preparing. A noteworthy preferred standpoint of the median filter over direct channels is that the median filter can take out the impact of info noise values with amazingly extensive extents. Middle channel is utilized for decreasing the measure of power variety between one pixel and the other pixel. In this channel, we don't supplant the pixel estimation of image with the mean of all neighboring pixel values, we replaces it with the middle esteem. At that point the middle is ascertained by first sorting all the pixel values into rising request and afterward supplants the pixel being computed with the center pixel [5]. On the off chance that the neighboring pixel of image which is to be considered contains an even quantities of pixels, than the normal of the two center pixel qualities is utilized to supplant. The middle channel gives best outcome when the motivation clamor rate is under $0.1 \%$. At the point when the amount of motivation commotion is expanded the middle channel not gives best outcome.

Advantage:

- It is anything but difficult to actualize.

- Used for de-noising distinctive sorts of clamors.

Disadvantage:

- Median Filter tends to remove image details while reducing noise such as thin lines and corners.

- Median filtering performance is not satisfactory in case of signal dependant noise. To remove these difficulties different variations of median filters have been developed for the better results.

\section{Related Work}

In this area diverse techniques are investigated to expel different sorts of noise from an image.

Claudio et.al [8] present a novel Hybrid Genetic Algorithm (HGA) for image de-noising, whose primary reason for existing is to reestablish pictures while protecting important data, for example, surface and edges. Hybrid technique joins administrators accessible in existing trans-formative strategies, for example, hybrid, and change and populace re-introduction with some best in class picture de-noising techniques. Trials are led on an arrangement of commotion sullied image regularly utilized by established researchers as benchmark, where distinctive levels of clamor are connected to the pictures.

Tao Dai et.al [9] present spatial or recurrence space without considering inter scale conditions. In this paper a novel fix based multistage items calculation (PMPA) for picture de-noising is proposed. It depends on fix likeness in spatial area and multistage items in wavelet space. PMPA is separated into two phases to prepare the smooth regions and non smooth zones, (for example, edges) exclusively. The main stage is in the wavelet space, then a locally versatile window based de-noising strategy (LAWML) in view of multistage items is connected to handle those wavelet coefficients relating to the non smooth regions, then get one beginning de-noised image. The second stage is in the spatial space, and afterward a non nearby means calculation is utilized to prepare those pixels in the smooth ranges to get another underlying denoised picture. The last de-noised image is gotten by a weighted averaging of every single normal pixel in both introductory de-noised images.

Licheng et.al [10] presents a straightforward and 
proficient particular sifting calculation (SFA) for expelling the motivation commotion in pictures. Coordinating the commotion finder with the connection between a pixel and its neighbors, the SFA can effectively recognize and evacuate clamor pixels while well safeguarding data pixels and examinations illustrate. It misleadingly fragments the pictures into three unique areas and afterward chooses a particular channel to evacuate the drive commotion in every district that the SFA beats a few existing de-noising strategies concerning the visual impacts and quantitative measure comes about. SFA calculation can productively evacuate the motivation clamor while well safeguarding the edges, splendid and dim ranges in pictures.

Jing Bai et.al [11] presents an enhanced square coordinating and 3D sifting (BM3D) in view of the setting is to diminish the clamor of low-dosage CT and enhance the picture quality. Facilitate, a visual consideration technique is utilized to highlight the injuries so that the unhealthy tissues can be enhanced in imaging contrast. The Contrast-to-commotion proportions (CNR) of 2 districts of-intrigue were enhanced from 0.8 and 0.7 to 1.2 and 2.5 , separately for two patients. The protest of this review is to de-noise the low-measurement CT and highlight the sores so that the specialists can recognize the injuries rapidly and adjust.

Kousuke et.al [12] presents a picture de-noising strategy utilizing non-neighborhood implies for a picture with Poisson clamor. The weighting capacity in the proposed technique conform the weight parameter in view of the evaluated clamor quality from the pixels in a nearby area. Therefore, the technique gives great clamor diminishment execution to Poisson commotion without plan of action to a fluctuation balancing out Transformation and exhibits that the clamor decrease of the proposed strategy is a change of 0.1-0.9 dB contrasted with the standard non-neighborhood implies. NLM technique diminishes the clamor as indicated by the commotion quality at every pixel utilizing the new weighting capacity. The PC reenactments showed that the commotion diminishment execution of the proposed strategy was enhanced by $0.1-0.9 \mathrm{~dB}$ in respect to that of the ordinary NLM.

Nelwin et.al [13] presents a picture de-noising utilizing shearlet change. They are ideally meager and have multiscale and multi-directional properties which are ideal in speaking to picture containing edges and examination of various limits that can be connected to the shear let change with a specific end goal to isolate loud coefficients from the first ones. The test comes about demonstrate that Otsu edge gives high PSNR and can be chosen as an ideal edge for picture de-noising.

Athira et.al [14] introduces non direct obscure parts, which have more than one movement segments. At that point, a visually impaired de-convolution procedure utilizing piecewise straight model is acquainted with gauge the obscure bits. Moreover, a de-noising method in light of wavelet multiform decay is assembled with this de-obscuring approach, keeping in mind the end goal to enhance the PSNR of the de-blurred pictures. The visually impaired de-convolution calculation utilizing piecewise straight guess alongside de-noising is viable recognizing the obscure parts introduce in the obscured pictures and can enhance the nature of estimation process. Besides, the proposed technique can enhance the PSNR estimations of the de-obscured pictures than ordinary de obscuring strategies

Xuefeng et.al[15] presents a pre-whitening system to the first HSI to change the clamor being a white one, then MWF can help to de-noise the pre-whitened information, at last a converse pre-whitening handling is utilized to modify the assessed flag. Near reviews with other denoising strategies demonstrate that this approach has promising prospects in this field. The capacity as a preprocessing calculation that enhances SVM and SAM characterization comes about connected to true HYDICE information. Quantitative outcomes in light of OA foundation assess the viability of this technique. The correlation with MWF, PCA and MNF licenses to value the de-noising effectiveness of our technique in the use of target grouping in uproarious HYDICE information

Lizhen et.al [16] presents stair casing impact of aggregate variety (TV) de-noising comes about, the high request distinction is regarded as the regularization thing in the high request total variation show (HOTV). The debasement rate of HOTV is speedier than the corruption rate of $\mathrm{TV}$ in the edge zone of the picture, so the edge subtle elements are lost in the HOTV show while the commotion is expelled. For lessening the stair casing impact and protecting the edge subtle elements viably, spatially versatile HOTV model is presented which distinction value is as enhanced versatile coefficient.

Jondhale et.al [17] show fix less Progressive Image De-noising (PID) is a dual domain picture de-noising strategy which continuously expels decrease the commotion from picture in every emphasis. It has straightforward usage utilizing strong commotion estimation and deterministic strengthening. Picture processing's applications like in protest following,

Yifeng et.al[18] presents restorative imaging, satellite imaging, confront acknowledgment and division requires picture de-noising as the preprocessing step and Issue with current picture de-noising techniques are blurring and antiques presents after expulsion of clamor from picture. Current de-noising strategies depend on patches of picture has well de-noising capacity yet implementation of such techniques are troublesome and conclusion that PID gives visual and hypothetical fabulous outcomes for both manufactured and regular Images.

Bansshidar et.al [19] presents a rebuilding plan to estimate the genuine picture debased by movement or out of center obscure together with added substance Gaussian clamor. The upper bound looking into it of regularization rouses picture de-noising before picture de-blurring. The clamor expulsion relies on the exact information of neighborhood insights. In like manner, a suitable neighborhood around every content pixel is chosen in light of the commotion 


\section{DE-NOISING ALGORITHMS}

\section{A. Hybrid Genetic Algorithm}

Jonatas[8] present Hybrid Genetic Algorithm (HGA) in view of the Genetic Algorithm (GA) presented, where an uproarious picture I is utilized as information and the populace is made by applying transformation administrators on the loud picture. Moreover, a portion of the hybrid administrators are the same presented. In any case, our work consolidates the GA, which is not the same as the GA utilized as a part with picture de-noising methods. Furthermore, an alternate wellness work controls the developmental procedure. Calculation 1 introduces a pseudo code that condenses the HGA working. HGA executes for a settled measure of time, where a similar populace develops while the best individual is not refreshed for $\max$ filter. When this measure is met, the populace is reset such that the best individual is kept and whatever remains of the new populace is made by a similar procedure connected to the underlying populace. Every individual is spoken to by a two-dimensional cluster of pixels with number values in the range $[0,255]$. A middle of the road populace is made amid the development venture of the HGA with people from the present populace and new people created [8]. These new people are made by hybrid administrators, whose two guardians are characterized by competition determination. Once the guardians are chosen, a hybrid administrator is chosen to make another person. Three sorts of hybrid administrators are utilized and they are arbitrarily picked each time another individual is created:

- One-point push: a column of pixels is arbitrarily picked. Every one of the pixels over this column will originate from one parent and every one of the pixels beneath this line will originate from the second parent.

- One-point segment: like the past technique, yet a section is picked as opposed to the column.

- Indicate point irregular: haphazardly picks every pixel from one of the guardians until the new individual is made.

The primary transformation administrator, in view of picture de-noising strategies, gives three beginning people that are de-noised pictures created by such techniques, where the best found is incorporated into the populace. In any case, to present differing qualities over the populace, the rest of the people are made by applying the second arrangement of change administrators over these three de-noised pictures. This introduction procedure happens in the start of the strategy to make the underlying populace and each time the populace is reinitialized.

\section{B. Patch Based Algorithm.}

Fix based calculations for picture de-noising has been broadly utilized as a part of late years. The greater part of fix based strategies simply misuse fix excess in spatial or recurrence area without considering interstate conditions. In this paper, we propose a novel fix based multistage items calculation (PMPA) for picture de-noising. Propose another fix based multi-scale items calculation (PMPA) joining patch comparability in spatial area and intra-andinterscale conditions in recurrence area. PMPA is separated into two phases to get two beginning de-noised pictures. The main stage is in the wavelet area, and we stretch out it to un-decimated wavelet space (UWT) and increase the nearby wavelet sub bands getting the multiscale items to misuse the wavelet inter-scale conditions. In addition, a locally versatile window-based de-noising strategy utilizing most extreme probability (LAWML) is then connected to handle those wavelet coefficients, comparing to non smooth districts, (for example, edges in spatial space), because of the straightforwardness and viability of LAWML[9]. One preferred standpoint of multi-scale items is that duplicating the contiguous wavelet sub bands that can upgrade edge structures while debilitating clamor. In the multi-scale items, huge structures, (for example, edges) can be successfully recognized from noise. Hence for every decay

Level of item sub band, a limit is figured in the multiscale items. From that point onward, a square window is utilized to perform traversal seeks in the multistate items in order to compute the neighboring vitality of items coefficients to recognize noteworthy highlights. In the wake of handling the wavelet coefficients by LAWML, we can get one starting de-noised picture by altering wavelet change. The second stage is in the spatial space, then we utilize a NL-implies strategy to handle those smooth pixels, comparing to smooth zones in the multiscale items, in the loud picture to misuse the fix excess specifically. In like manner, we can acquire another introductory de-noised picture by the NL-implies technique. Along these lines, the last de-noised picture is acquired by a weighted averaging of all normal pixels in both introductory de-noised pictures. It depends on fix comparability in spatial space and multistage items in wavelet area. PMPA is isolated into two phases to prepare the smooth territories and non smooth zones, (for example, edges) exclusively [10]. The primary stage is in the wavelet space, then a locally versatile window based de-noising technique (LAWML) in light of multistage items is connected to prepare those wavelet coefficients relating to the Non smooth regions, then acquire one beginning de-noised picture. The proposed PMPA can achieve better results for those images containing fewer textures, such as Lena and Peppers. In this manner the execution of PMPA can be additionally enhanced by developing the inquiry window measure, in any case, at the cost of computational cost, so we restrain the hunt window size to a settled esteem, which is an exchange off between the execution and computational many-sided quality. Consequently PMPA is more reasonable for piecewise smooth pictures because of the property of NLmeans [10]. The human eye is the just a single to choose whether the nature of the pictures has been enhanced by the de-noising calculation. We show some de-noising comes about contrasting the PMPA and other state of- 
the-workmanship de-noising techniques in Figure 3. From Figure 3, we can understand that the proposed PMPA have preferable visual impacts over BLSGSM and NL-implies, which have all the more ringing antiques and more subtle edges, separately. Contrasted and BM3D, both strategies can evacuate the greater part of clamor adequately, notwithstanding, the PMPA can have better visual impacts in smooth zones. With respect to the time many-sided quality, the proposed de-noising calculation is like BM3D, higher than BLS-GSM and NL-implies. The introduced technique and BM3D show signs of improvement comes about by giving up a few measure of calculation time to get better exhibitions.

\section{Selective Filtering Algorithm for Image De-noising}

Selective filtering Algorithm (SFA) for evacuating the motivation clamor in pictures. Coordinating the clamor finder with the connection between a pixel and its neighbors, the SFA can proficiently distinguish and expel commotion pixels while well protecting data pixels. SFA is to select a suitable channel to evacuate commotion in various locales inside a picture. This channel determination changes adaptively as indicated by the picture substance [11]. SFA first sections the data picture into the dull, breathtaking and dim locale. By then, every pixel is consigned to one of these three regions. The WMF, HMF, and CHMF are then decided for removing hullabaloo in diminished, dull and astonishing Regions, separately. It incorporates three sorts of great mean channels, to be specific the weighted, symphonious, and contra consonant

Table 1. PSNR Performance Measure and Experiment Results of Various De-Noising Algorithm [8], [9], [10].

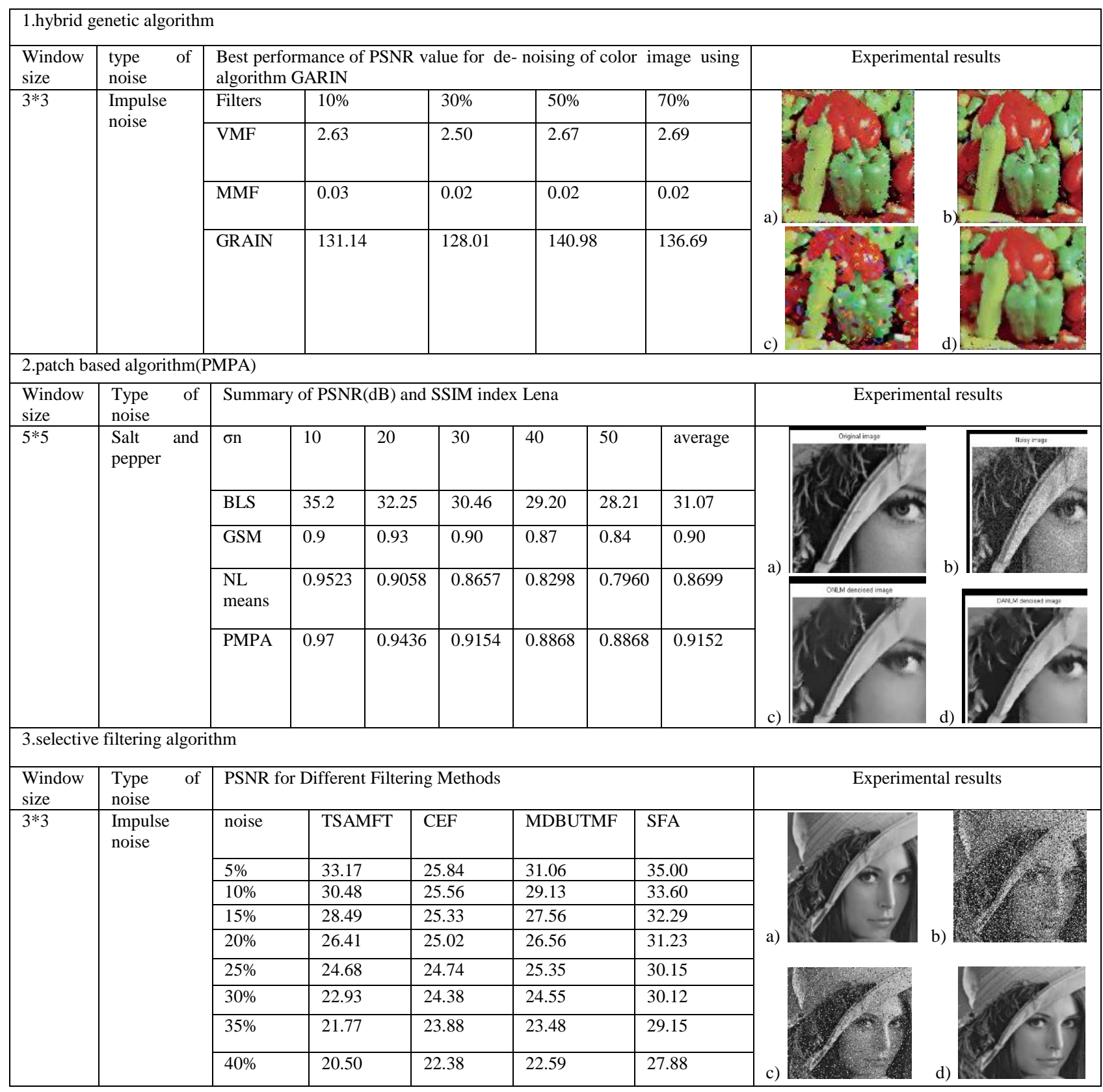


Mean channels. As indicated by SFA, the calculation adaptively picks a suitable channel for evacuating the drive clamor in various areas inside a picture. Utilizing a few straightforward customary channels, the SFA yields promising de-noising comes about which are superior to the several state of human expressions. SFA first fragments the information picture into the dim, splendid and dim areas. At that point, every pixel is doled out to one of these three areas. The WMF, HMF, and CHMF are then chosen for expelling clamor in the dim, dull and splendid districts, separately. SFA presents a novel specific separating calculation (SFA) for picture denoising. Since picture substance may change fundamentally in various areas inside a picture, one single de-noising channel for the entire picture might be do not fitting anymore. The hidden major of the proposed SFA is to choose a suitable channel to expel commotion in various locales inside a picture [11], [12]. This channel choice changes as indicated by the picture substance. SFA calculation in each picture of dim scale level is of power 0 to 255 . The SFA likewise treats the salt and pepper commotions independently and salt clamor (white spots) is additionally irritating in the dull locales in a picture, the HMF, which is great at evacuating the salt commotion, is used; On the other hand, in light of the fact that the pepper commotion (dark dabs) is all the more disgusting in the brilliant districts, the CHMF that can well smother the pepper clamor is then utilized in the proposed SFA. SFA expel the drive clamor operating at a profit and brilliant districts in a picture while saving the data pixels. SFA has been connected to different dim scale pictures with various components to evaluate its denoising execution. Sifting low tainted pictures is all the more intriguing in light of the fact that it requires exact recognition of adulterated pixels to save subtle elements and edges in pictures. SFA had been contrasted and a few existing sifting strategies, for example, the resilience based particular number juggling mean separating strategy (TSAMFT) differentiate Upgrade based channel (CEF) and adjusted choice based unsymmetrical trimmed middle channel (MDBUTMF).The SFA moreover treats the salt and pepper clatters freely. See that the salt uproar (white spots) is all the all the more aggravating negligent areas in a photo, the HMF, which is awesome at emptying the salt tumult, is utilized [12]. On the other hand, in light of the way that the pepper hullabaloo (dull bits) is also horrible in the splendid regions [19], the CHMF that can well cover the pepper clatter is then used in the proposed SFA. Thusly, this new SFA can remove the drive tumult working at a benefit and mind blowing ranges in a photo.

\section{CONCLUSION}

Distinctive filtering procedures and de-noising calculation are utilized for noise lessening in an image. Besides, we displayed and thought about outcomes for these sifting procedures. The outcomes acquired utilizing half breed hereditary calculation additionally called Hybrid genetic algorithm guarantees commotion free, which takes as info a loud image and produces as yield a diminished noise rendition of a similar image. As a component of its developmental procedure, hybrid incorporates the execution of powerful and versatile channels from writing with the mean to join and enhance their outcomes. The fundamental favorable circumstances of this cross breed hereditary calculation are the denoising ability of the demolished shading part contrasts. A critical favorable position of hybrid genetic algorithm is its adaptability, this preferred standpoint could be extrapolated to a more broad arrangement, considering $n$ sorts of clamor, $\mathrm{n}$ channels and $\mathrm{m}$ commotion densities. This is likewise left as an objective for future work.

\section{REFERENCES}

[1] “A NEW SELECTIVE FILTERING ALGORITHM FOR IMAGE DENOISING Licheng Liu , Yicong Zhou *, and C . L . Philip Chen Department of Computer and Information Science, University of Macau," pp. 193-197, 2013.

[2] W. H. W. Sduw et al., “, PDJH GHQRLVLQJ DOJRULWKP EDVHG RQ VWUXFWXUH DQG,” pp. 147-151, 2016

[3] J. L. De Paiva, S. Carlos, S. Paulo, S. Carlos, and S. Paulo, "A Hybrid Genetic Algorithm for Image Denoising," pp. 2444-2451, 2015.

[4] M. C. Mythili and D. V. Kavita, "Efficient Technique for Color Image Noise Reduction," Res. Bull. Jordan ACM, vol. 2, no. 3, pp. 41-44, 2011.

[5] V. Murugan and T. Avudaiappan, "A Comparative Analysis of Impulse Noise Removal Techniques on Gray Scale Images," vol. 7, no. 5, pp. 239-248, 2014.

[6] E. J. Leavline, D. A. Antony, and G. Singh, "Salt and Pepper Noise Detection and Removal in Gray Scale Images: An Experimental Analysis," vol. 6, no. 5, pp. 343-352, 2013.

[7] A. Kumar Boyat and B. K. Joshi, "a Review Paper: Noise Models in Digital Image Processing," An Int. J., vol. 6, no. 2, pp. 63-75, 2015.

[8] J. L. De Paiva, S. Carlos, S. Paulo, S. Carlos, and S. Paulo, "A Hybrid Genetic Algorithm for Image Denoising," pp. 2444-2451, 2015.

[9] T. Dai, C. B. Song, J. P. Zhang, and S. T. Xia, "PMPA: A patch-based multiscale products algorithm for image denoising," Proc. - Int. Conf. Image Process. ICIP, vol. 2015-Decem, no. 1, pp. 4406-4410, 2015.

[10] “A NEW SELECTIVE FILTERING ALGORITHM FOR IMAGE DENOISING Licheng Liu, Yicong Zhou *, and C . L . Philip Chen Department of Computer and Information Science, University of Macau," pp. 193-197, 2013.

[11] L. L. Chen, S. P. Gou, Y. Yao, J. Bai, and L. Jiao, "Based BM3D," pp. 682-685, 2016.

[12] K. Imamura, N. Kimura, F. Satou, S. Sanada, and Y. Matsuda, "Image Denoising using Non-local Means for Poisson Noise," pp. 1-6, 2016.

[13] T. S. Anju and N. R. N. Raj, "Denoising of digital images using shearlet transform," 2016 IEEE Int. Conf. Recent Trends Electron. Inf. Commun. Technol., no. 1, pp. 893896, 2016.

[14] N. R. N. R and A. S. Vijay, "Adaptive Blind Deconvolution and Denoising of Motion Blurred Images," pp. 1171-1175, 2016.

[15] X. Liu, Y. Feng, Y. Li, and M. Fu, "Denoising Hyperspectral Images with Non-White Noise Based on 
Tensor Decomposition," no. 1.

[16] L. Deng, Q. Fang, and H. Zhu, "Order Total Variation Model," no. 61501259, pp. 212-216, 2016.

[17] K. C. Jondhale, "Improved Denoising Technique for Natural and Synthetic Images," no. I, pp. 1-4.

[18] W. H. W. Sduw et al., ", Image denoising algorithm based on structure and texture part," pp. 147-151, 2016.

[19] K. Xpdu et al., "\$A Denoising inspired d debluring framework for regularized image restoratin," vol. 6, pp. 3-8.

\section{Authors' Profiles}

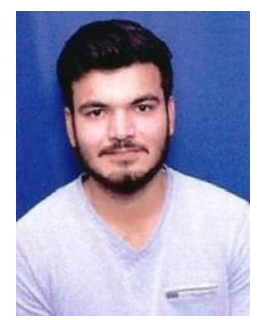

Gourav is currently persuing M.tech in computer science from CGC-COE, Mohali, Punjab. He received his B.tech degree in computer science from S.S.C.E.T Badhani, Punjab in 2014 and presently working in the area of "digital image processing" with his Asst. professor Tejpal Sharma.

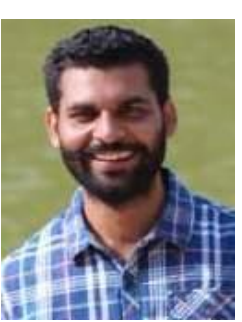

Tejpal Sharma presently persuing Ph.d on "digital image processing" from Punjab university, Chandigarh and receive his M.tech degree from B.B.S.B Engineering College, Fatehgarh Sahib, Punjab. Currently working as an Assistant professor in Chandigarh group of College, Landran, Mohali and Punjab

How to cite this paper: Gourav, Tejpal Sharma,"Various Types of Image Noise and De-noising Algorithm", International Journal of Modern Education and Computer Science(IJMECS), Vol.9, No.5, pp. 50-58, 2017.DOI: 10.5815/ijmecs.2017.05.07 